\title{
COMPARISON AND ANALYSIS OF ORTHOGONAL AND BIORTHOGONAL WAVELETS FOR ECG COMPRSSION
}

\author{
Ravi Shankar Meena ${ }^{1}$, Ambalika Sharma ${ }^{2}$ \\ ${ }^{1}$ M.Tech, Department of Electrical Engineering, Indian Institute of Technology Roorkee, Uttrarakhand, India \\ ${ }^{2}$ Assistant Professor, Department of Electrical Engineering, Indian Institute of Technology Roorkee, Uttrarakhand, India
}

\begin{abstract}
In the present work we analyze the performance of orthogonal and Biorthogonal wavelet for electrocardiogram (ECG) compression. Both types of wavelets applied on different types of records taken from MIT-BIH database. A thresholding algorithm based on energy packing efficiency of the wavelet coefficients is used to threshold the wavelet coefficients. We divide wavelet coefficients in two groups approximation coefficients group and detail coefficients group then, select an appropriate threshold value for each detail sub band according to the energy packing efficiency of the each sub band. We have implemented different types of wavelets for the compression of the ECG signal and compared their performance. We have evaluated the effect of decomposition levels on compression ratio (CR) and preserved energy. We measured CR and percentage root mean square difference (PRD) and it is found that Bi orthogonal wavelet performs well than the orthogonal ones in both the criteria.
\end{abstract}

Keywords: ECG compression; wavelet transform; energy packing efficiency; PRD

\section{INTRODUCTION}

Electrocardiogram signal is very useful for the diagnosis of different types of heart abnormalities. Main advantage in the measurement of ECG signal is that it can be easily measured with the help of the surface electrodes, we need not to be penetrate the electrodes in the body. In present time, we need to transmit the ECG signal to the remote places for the better diagnosis [1].

The main aim of any compression algorithm is to get maximum data reduction with preserving the remarkable clinical information of the original signal. Many tools are available for the efficient compression of ECG signal such as Fourier transform, Discrete cosine transform, Wavelet transform. Wavelet transform is one of the promising tool for the ECG compression [2]. It has received significant attention recently due to it's performance in ECG compression [3]. The basic parameters for measuring compression performance are compression ratio, PRD and preserved energy.

In the present work, thresholding technique based on the energy packing efficiency (EPE) [4] When discrete wavelet transform (DWT) applied on the ECG signal, it decomposes the signal into multiresolution bands, a appropriate threshold value is selected for each band of the signal according to the EPE is measured, then calculate compression ratio and PRD. We analyze different types of wavelet families for the compression of the different ECG records and then compare the performance of the wavelets. We also analyzed the effect of decomposition level of wavelets on the compression and preserved energy .This paper is organized as follows: Section II presents a brief introduction of wavelet transform. Section III and IV presents compression algorithm used and results, respectively.

\section{WAVELET TRANSFORM}

Wavelet transform is used to analyze the time as well as frequency domains signals. It is suitable for the analysis of the time-varying non stationary signals such as ECG because they provide different frequency resolution bands after decomposition process instead Fourier and Short Time Fourier Transform (STFT). Fourier Transform provides constant frequency response so it is difficult to analyze non-stationary signals and STFT provides Fourier Transform of the function at some instant of time $t$, as examined as some time limited window center at time t. Window's width of the STFT is constant so it does not provide multi resolution analysis of the signal [5]. Wavelet transform provides both frequency as well as time resolution. A mother wavelet $\psi(t)$ is a function of zero average:

$$
\int_{-\infty}^{\infty} \psi(t) d t=0
$$

If this function is dilated by scalar ' $a$ ', and translated by another scalar ' $b$ ' then mother wavelet can be represented by

$$
\psi_{\mathrm{a}, \mathrm{b}}(\mathrm{t})=\frac{1}{\sqrt{\mathrm{a}}} \psi\left(\frac{\mathrm{t}-\mathrm{b}}{\mathrm{a}}\right)
$$


Wavelet transform $\mathrm{X}(\mathrm{a}, \mathrm{b})$ of signal $\mathrm{x}(\mathrm{t})$ is given by:

$$
X(a, b)=\frac{1}{\sqrt{a}} \int_{-\infty}^{\infty} x(t) \psi\left(\frac{t-b}{a}\right) d t
$$

Digital representation of (3) is known as Discrete Wavelet Transform (DWT):

$$
\operatorname{DWT}(m, k)=\frac{1}{\sqrt{a^{m}}} \sum_{k=0}^{N} x(k) \psi\left(a^{m} n-k\right)
$$

DWT is based on the sub band coding is found to yield a fast computation of wavelet transform [6]. It is easy to implement and reduce the time and computations required. The DWT is computed by successive low pass and high pass filtering of the digital domain signal.

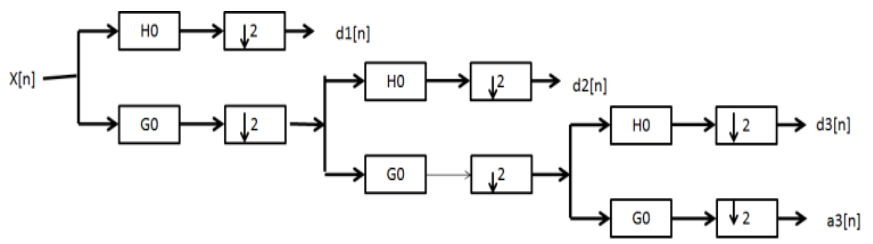

Fig -1: Discrete Wavelet Transform

In the figure, signal is denoted by $\mathrm{x}[\mathrm{n}]$, where $\mathrm{n}$ is a integer. The low pass filter is denoted by G0 while high pass filter is denoted by H0. At each level, high pass filter produces detail information $\mathrm{d}[\mathrm{n}]$, while low pass filter associated with the scaling function produces coarse approximations $\mathrm{a}[\mathrm{n}]$.

In this paper, it is used a DWT with the decomposition level L ( $\mathrm{L}=7$ and $\mathrm{L}=3)$, length $\mathrm{N}(\mathrm{N}=2000)$ of the signal.

\subsection{Wavelet Families}

There are a number of basic functions used as mother wavelet for the wavelet transformation, but wavelet families can be divided into two main categories, orthogonal and Biorthogonal wavelets [5]. In orthogonal wavelets analyzing and synthesis filters have same length but in Biortohogonal wavelets both can be different length.

\section{ECG DATA COMPRESSION ALGORITHM}

In the compression algorithm, First Wavelet Transform is applied on the ECG signal consisting $\mathrm{N}$ samples. Wavelet transform divides the signal into multiresolution sub bands. Calculate energy of each band then an appropriate threshold value is selected for each band such as preserved energy after the reconstruction of the original signal retain up to a remarkable value. Then, the wavelet coefficients are threshold with the determined threshold value for each sub bands. Then, signal is reconstructed with the help of the significant coefficients. Now calculate compression ratio and PRD of the signal.
As we know every transformation technique is lossy compression technique so this technique is also loosed some redundant information at the time of compression. We can calculate the retaining information with the help of preserved energy (EPE) in the reconstructed signal. In the following subsections, detailed descriptions of the ECG compression algorithm are given.

\subsection{Wavelet Transform of ECG Signal}

The ECG signal is decomposed by the wavelet transform into multiresolution sub bands. In the present work, ECG signal is decomposed by the different types of wavelets with different decomposition levels because in [7] it has been pointed out that compression is affected by the decomposition levels, so for the comparison purpose we decomposed the signal up to third and seventh level.

\subsection{Thresholding Algorithm}

- After the decomposition of the original signal using wavelet transform into different sub bands, an appropriate threshold value or level is required to threshold the wavelet coefficients as well as to control the CR and PRD.

- The choice of the threshold value is a difficult task because a large threshold value can reduce large amount of data but energy retained in the reconstructed signal or quality of reconstructed signal is poor. On the other hand, a small threshold value provides low data reduction but high quality of the reconstructed signal.

- In the present work, we calculate the energy packing efficiency of each subband. Wavelet decomposes the signal into multiresoution sub band, the approximation band (lowest resolution band) contains high amplitude coefficients and it is smallest in size. The detail bands, other than the those of the approximation band contains small magnitude coefficients and they can be discarded in the case of lossy compression of the medical signals because they have low amplitude coefficients or energy.

- The energy contribution of each detail sub band to the whole decomposition sub bands has been calculated by energy packing efficiency (EPE) [6]. In this case EPE is the percentage quality that represents the total preserved energy of certain decomposed sub band after thresholding with respect to the total energy in that sub band before thresholding and is defined as

$$
\operatorname{EPE}(\%)=\frac{\sum_{n=1}^{M i}(c(n))^{2}}{\sum_{n=1}^{M}(c(n))^{2}} \quad \mathrm{x} 100
$$

Where Mi and M are the number of coefficients in the $\mathrm{i}^{\text {th }}$ sub band after thresholding and the whole number of coefficients in that sub band before thresholding respectively 
To calculate the energy contribution of each subband after decomposition with respect to the whole number of wavelet coefficients.The wavelet transform was applied to decompose first 2000 samples of the MITBIH data base record 101 up to seventh level . Results of EPE of different subbands shown in Table 1. We can see that the energy contribution of approximation coefficients (26) sub band to the total energy is $89.13 \%$ and the energy contribution of detail coefficients (2048) sub bands to the total energy is $10.87 \%$.

Table -1: EPE values of the Different bands afterDecomposition for Record 101

\begin{tabular}{|l|l|}
\hline $\begin{array}{l}\text { Symbol of EPE for } \\
\text { the sub bands }\end{array}$ & $\begin{array}{l}\text { Values of EPE in the respective } \\
\text { sub band (\%) }\end{array}$ \\
\hline EPED1 & .01209 \\
\hline EPED2 & 2.422 \\
\hline EPED3 & 2.0337 \\
\hline EPED4 & 1.1500 \\
\hline EPED5 & 1.8604 \\
\hline EPED6 & 2.539 \\
\hline EPED7 & 0.8166 \\
\hline EPEA7 & 89.13 \\
\hline
\end{tabular}

- Based on this analysis we have applied following thresholding rule:

First, divide the subbands in two groups : 1) approximation band coefficients (A7) and, 2) the whole detail band coefficients (D7-D1). The approximation band kept without thresholding and we have selected the threshold value for each detail sub band. In order to select the threshold value for each detail subband, coefficients of each detail subband is sorted in descending order and range to be checked of high energy (information) contain coefficients according to the figure (2) shown in each detail sub band, then a appropriate threshold level is selectedand calculate CR and PRD for the signal.

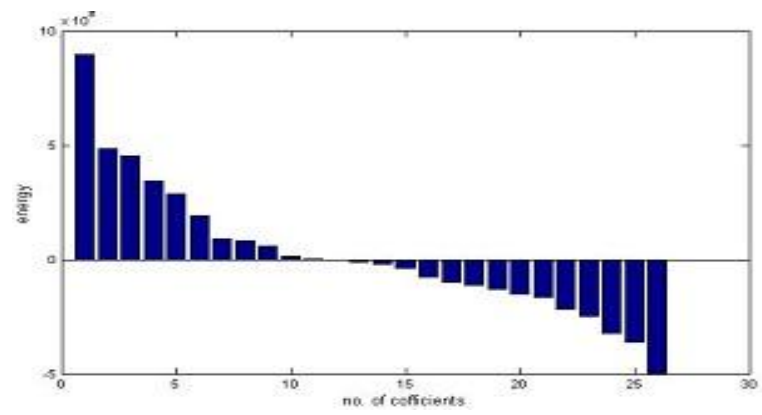

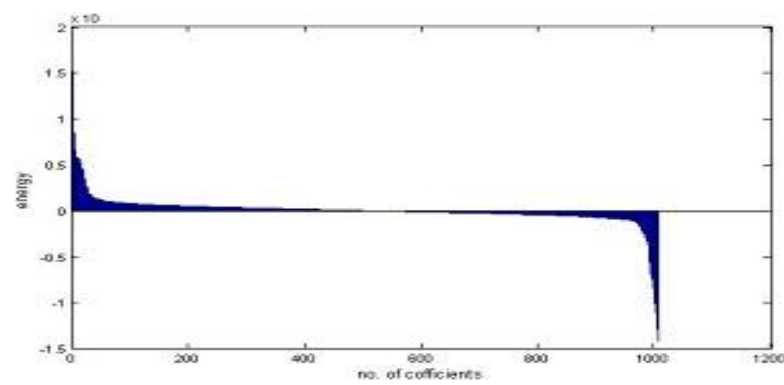

Fig -2:Energy level of D7 and D1 sub bands cofficients after sortig respectively, for Record 101

\section{PERFORMANCE AND ANALYSIS}

In this section, the proposed algorithm is tested using the MITBIH database which consists 48 hour ECG records. Results of different wavelet families is compared and analyzed using the well known parameters compression ratio, PRD and preserved energy in the reconstructed signal.We have used Daubechies (db5, db3), Biortogonal wavelets (Bior5.5, Bior3.3) and Coiflet (coif5, coif3) at two different decomposition levels (3rd, 7th). The record numbers for the database used are: 100, 101, 103, 104, 105, 106, 107, 108, 109, 117, 119.

\subsection{Performance Parameters}

Compression ratio and $\mathrm{PRD}$ two parameters are used to measure the performance. The compression ratio is defined as:

$$
\mathrm{CR}=S / C
$$

Where, $\quad \mathrm{S}-$ total no. of wavelet coefficients $\mathrm{C}-$ no. of significant wavelet coefficients after thresholding

The PRD is calculated using:

$$
P R D=\sqrt{\frac{\sum_{n=1}^{N}(x(n)-x r(n))^{2}}{\sum_{n=1}^{N}(x(n))^{2}}}
$$

Where, $x(n)$ and $x \mathrm{r}(n)$ are the original and reconstructed signal, respectively, and $\mathrm{N}$ is the length of the window over which the PRD is calculated.

\subsection{Simulation Results}

For the comparison purpose, the proposed algorithm is applied to the same data sets with different wavelet families (bior, $\mathrm{db}$, coif) as well as at different decomposition levels. Results of comparison and analysis of different wavelets are shown in the tables and figures given below. 
Table -2: CR and PRD values for Different data sets for 'bior' wavelet family up to $7^{\text {th }}$ Decomposition level

\begin{tabular}{|l|l|l|l|}
\hline Records & CR & PRD\% & EPE\% \\
\hline 100 & 7.77 & 3.64 & 91.45 \\
\hline 101 & 6.66 & 6.62 & 93.00 \\
\hline 103 & 8.01 & 5.29 & 75.28 \\
\hline 104 & 6.34 & 3.57 & 75.14 \\
\hline 105 & 7.03 & 6.88 & 88.13 \\
\hline 106 & 6.23 & 4.81 & 90.94 \\
\hline 107 & 4.6 & 7.5 & 83.77 \\
\hline 108 & 6.82 & 5.85 & 86.44 \\
\hline 109 & 4.57 & 6.11 & 88.88 \\
\hline 117 & 4.52 & 5.6 & 87.92 \\
\hline 119 & 4.63 & 7.42 & 72.24 \\
\hline
\end{tabular}
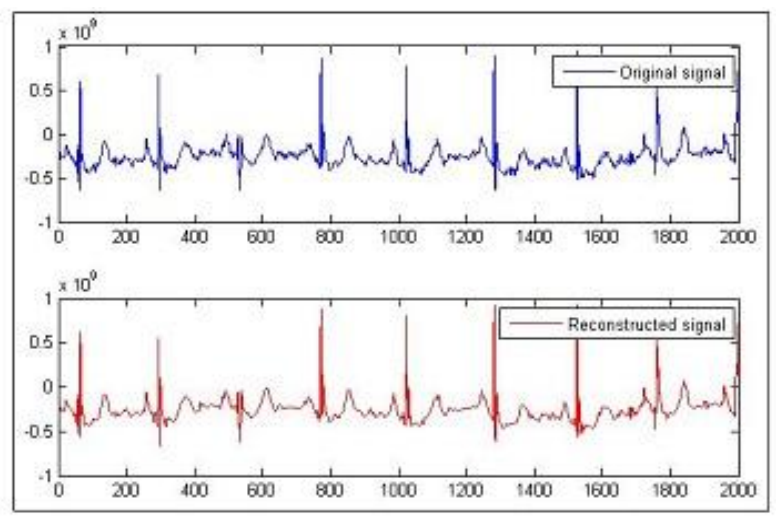

Fig-3:Original and Reconstructed signal for biorwavelet family at $7^{\text {th }}$ Decomposition level

We used different record taken from MIT-BIH data base and applied different types of wavelet families (bior, db, coif). Results shown in the Table 2 to 4 . Original and reconstructed signal for record 101 using bior wavelets is shown in Fig.3.

Results of effect of different decomposition levels are shown in Table 5 to 7. The comparison analysis of preserved energy (EPE) of the reconstructed signal using wavelet families (bior, $\mathrm{db}$, coif) at the same $\mathrm{CR}$ is shown in Fig. 4 up to 7 th decomposition level and effect of increasing decomposition level (3rd and 7th) on compression ratio is shown in Fig. 5 for the record 101.

Table -3:CR and PRD for Different data sets for 'db' wavelet family up to $7^{\text {th }}$ Decomposition level

\begin{tabular}{|l|l|l|l|}
\hline Records & CR & PRD\% & EPE\% \\
\hline 100 & 7.31 & 12.27 & 88.06 \\
\hline 101 & 6.59 & 29.49 & 89.49 \\
\hline 103 & 7.75 & 9.98 & 64.47 \\
\hline
\end{tabular}

\begin{tabular}{|l|l|l|l|}
104 & 6.11 & 11 & 64.56 \\
\hline 105 & 6.8 & 19.79 & 85.69 \\
\hline 106 & 6.26 & 12.42 & 81.38 \\
\hline 107 & 4.56 & 13.68 & 77.83 \\
\hline 108 & 6.69 & 13.43 & 83.40 \\
\hline 109 & 4.52 & 13.95 & 84.76 \\
\hline 117 & 4.52 & 16.5 & 92.19 \\
\hline 119 & 4.52 & 19.25 & 70.52 \\
\hline
\end{tabular}

Table -4:CR and PRD for Different data sets for ' $\mathrm{db}$ ' wavelet family up to $7^{\text {th }}$ Decomposition level

\begin{tabular}{|l|l|l|l|}
\hline Records & CR & PRD $\%$ & EPE\% \\
\hline 100 & 7.77 & 44.81 & 91.13 \\
\hline 101 & 6.76 & 26.22 & 92.14 \\
\hline 103 & 7.94 & 36.8 & 72.59 \\
\hline 104 & 6.37 & 36.79 & 70.63 \\
\hline 105 & 6.98 & 44.06 & 91.14 \\
\hline 106 & 6.47 & 30.96 & 94.46 \\
\hline 107 & 4.85 & 55.31 & 85.83 \\
\hline 108 & 6.66 & 47.96 & 87.90 \\
\hline 109 & 4.66 & 40.26 & 92.18 \\
\hline 117 & 4.77 & 40.01 & 94.89 \\
\hline 119 & 4.66 & 66.16 & 72.41 \\
\hline
\end{tabular}

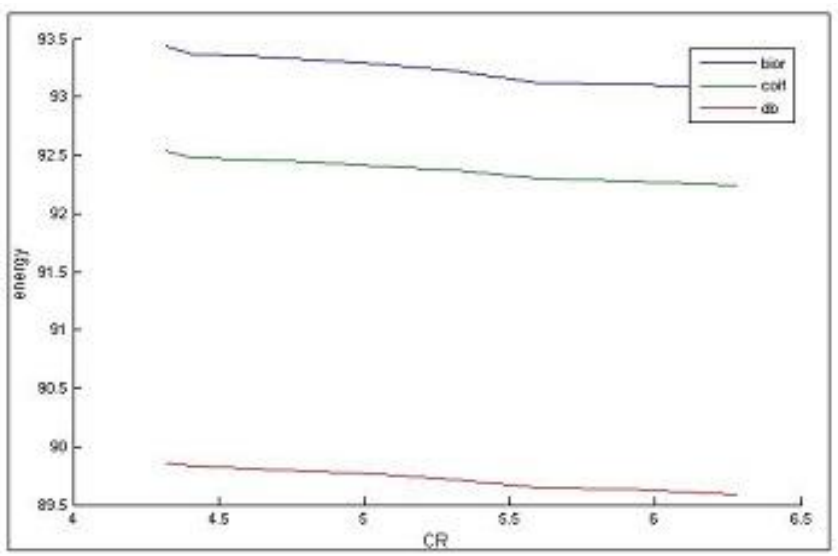

Fig-4:Energy comparison between bior,db and coifletwavelet families respectively, at the same CR up to 7th level decomposition 


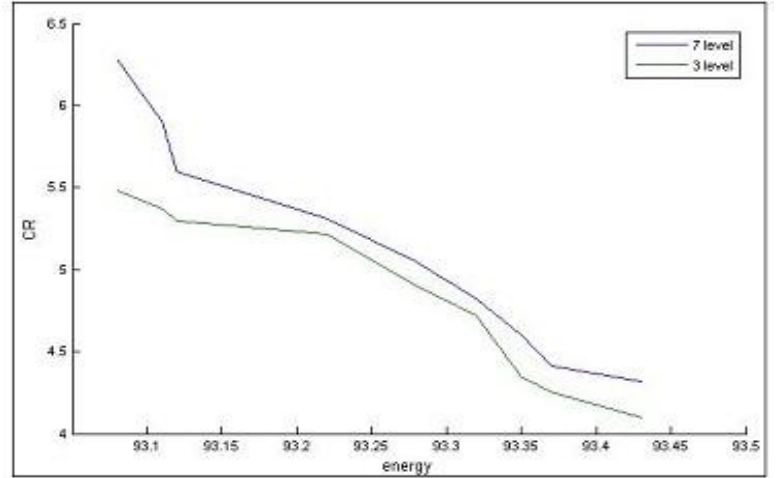

Fig-5:CR comparison at the same preserved energy level for bior wavelet family at $7^{\text {th }}$ and $3^{\text {rd }}$ level respectively

The results shows that Biorthogonal wavelet provides better compression performance comparatively other two wavelets used. The preserved energy in the reconstructed signal is also better in Biorthgonal wavelet results. The PRD value is also small in Biorthogonal results so very less diagnostic information is loosed comparatively other two wavelet families. Reason behind this performance is that biorthogonal wavelets can use both similar and dissimilar order for the decomposition.

In our analysis, we also found that the effect of decomposition levels It is also found that (Table. 2 to 7), if we kept EPE of the reconstructed signals is constant at 7th and 3rd level respectively, then compression ration decreases at the $3 \mathrm{rd}$ decomposition level for all three wavelet families.

It is also found that value of PRD is high and compression ratio is also low when 'coif' wavelets is used but EPE values of reconstructed signals is same as' bior' wavelets. So different wavelet families can be used depends on the requirements of compression.

Table-5:CR and PRD for the Different data sets for 'bior' wavelet family up to 3rd Decomposition level

\begin{tabular}{|l|l|l|l|}
\hline Records & CR & PRD\% & EPE\% \\
\hline 100 & 5.64 & 9.12 & 91.45 \\
\hline 101 & 5.59 & 8.41 & 93.00 \\
\hline 103 & 3.97 & 13.21 & 75.28 \\
\hline 104 & 5.06 & 15.33 & 75.14 \\
\hline 105 & 5.44 & 12.61 & 88.13 \\
\hline 106 & 5.64 & 9.75 & 90.94 \\
\hline 107 & 4.31 & 15.70 & 83.77 \\
\hline 108 & 4.23 & 12.33 & 86.44 \\
\hline 109 & 4.26 & 10.45 & 88.88 \\
\hline 117 & 3.85 & 11.57 & 87.92 \\
\hline 119 & 4.27 & 25.04 & 72.24 \\
\hline
\end{tabular}

Table-6:CR and PRD for the Different data sets for ' $\mathrm{db}$ ' wavelet family up to $3^{\text {rd }}$ Decomposition level

\begin{tabular}{|l|l|l|l|}
\hline Records & CR & PRD\% & EPE\% \\
\hline 100 & 5.48 & 11.27 & 88.06 \\
\hline 101 & 5.46 & 9.90 & 89.49 \\
\hline 103 & 5.81 & 22.22 & 64.47 \\
\hline 104 & 4.98 & 20.30 & 64.56 \\
\hline 105 & 5.74 & 13.74 & 85.69 \\
\hline 106 & 6.05 & 13.83 & 81.38 \\
\hline 107 & 4.17 & 18.87 & 77.83 \\
\hline 108 & 5.49 & 11.58 & 83.40 \\
\hline 109 & 5.33 & 12.44 & 84.76 \\
\hline 117 & 3.53 & 7.90 & 92.19 \\
\hline 119 & 4.46 & 26.72 & 70.52 \\
\hline
\end{tabular}

Table-7:CR and PRD for the Different data sets for 'coif' wavelet family up to 3rd Decomposition level

\begin{tabular}{|l|l|l|l|}
\hline Records & CR & PRD\% & EPE\% \\
\hline 100 & 5.50 & 8.05 & 91.13 \\
\hline 101 & 5.60 & 7.35 & 92.14 \\
\hline 103 & 3.10 & 9.53 & 72.59 \\
\hline 104 & 5.10 & 18.49 & 70.63 \\
\hline 105 & 5.29 & 9.36 & 91.14 \\
\hline 106 & 5.12 & 6.04 & 94.46 \\
\hline 107 & 4.32 & 13.54 & 85.83 \\
\hline 108 & 4.55 & 10.03 & 87.90 \\
\hline 109 & 3.72 & 8.36 & 92.18 \\
\hline 117 & 3.06 & 5.03 & 94.89 \\
\hline 119 & 4.19 & 25.63 & 72.41 \\
\hline
\end{tabular}

\section{CONCLUSIONS}

For the compression of single dimension signals this algorithm is proposed. An appropriate threshold value is selected for the each detail sub band based on the EPE. Then, we thresholded the coefficients of the sub bands and reconstruct the signal with help of the significant coefficients after thresholding. The algorithm is tested on 15 ECG signals taken from MIT-BIH data base.

Biorthogonal wavelets provides better compression performance but it is found that it requires more computational efforts than Daubechies wavelets. It is also found that Biorthogonal wavelets take more time than other wavelet families [7].

In study, we also analyzed that effect of decomposition levels on compression performance. If decomposition levels increased then compression performance is better but signal quality is poor. 


\section{ACKNOWLEDGEMENTS}

I would like to thank my friend Chandrasekhar Shithole for his help in MATLAB programming and suggestions given to me at different stages of my work.

\section{REFERENCES}

[1]. L. Cromwell andF. J. Weibell, E. A. Pfeiffer, "Biomedical Instrumentation and Measurements" 2nd Edition, Prentice Hall, Inc.

[2]. A. A. Shinde, "The Comparison of Different Transform Based Methods for ECG Data Compression, " in ICSCCN, 2011

[3]. B. A. Rajoub. "An Efficient Coding for the Compression of ECG Signals Using the Wavelet Transform", IEEE Trans. on Biomed. Engineering, vol 49, pp, April, 2002.

[4]. M. S. Hossain, T. Aziz and M. A. Haque, "ECG Compression Using Multilevel Thresholding of Wavelet Coefficients," in ISSNIP, pp. 321- 326, 2008

[5]. A. Ebrahimzadeh and M.Azarbad, "ECG Data Compression Using Wavelet Transform and Three-Level Quantization,"in IDC, pp. 250-254, 2010

[6]. L. A. Barford, R. S.Fazzio and D, R, Smith "An Introduction to Wavelets," Instruments and Photonics Laboratory, Hewlett-Packard Company, Sep 1992.

[7]. K. Lees, "Image Compression using Wavelets," May, 2002.

[8]. Andrés E. Gaona and Luis F. Pedraza, "Discrete Wavelet Transform in Compression and Filtering of Biomedical Signals" University Francisco José de Caldas, Colombia, 2011

[9]. A. Sandryhaila, ,S. Saba, M. Puschel and J. Kovacevic, "Efficient Compression of QRS Complexes Using Hermite Expansion," IEEE Trans. Signal Processing, vol. 60, pp. 947955, Feb-2012.

[10]. M. S. Manikandan and S. Dandapat, "Wavelet based EGG Compression with Large Zero Zone Quantizer," in India Conference Annual IEEE, pp. 1-5, 2006.

[11]. S. Malik and V. Verma, "Comparative analysis of DCT, Haar and Daubechies Wavelet for Image Compression," in ISSN, vol. 7, 2012.

[12].E. Berti, F. Chiaraluce, N. E. Evans, and J. J. McKee," Reduction of Walsh-Transformed Electrocardiograms by Double Logarithmic Coding," IEEE Trans On Biomed Engineering, vol. 47, pp- Nov. 2000.

[13]. S. Rout, "Orthogonal and Biorthogonal Wavelets for Image Compression," Blacksburg, Virginia, 2003.

[14]. A V. Chagas, EAB Da Silva, J. Nadal,"ECG Data Compression Using Wavelets," Computer in Cardiology, vol. 27, pp. 423-426, 2000.

[15]. C. T. Ku, K. C. Hung, T. C. Wu and H.S. Wang, "Wavelet Based ECG Data Compression With Linear Quality Control Sheme," IEEE Trans. Biomed. Engineering, vol. 57, pp. 1399-1409, 2010 